\title{
Proceeding
}

Supplementary Issue: Spring Conferences of Sports Science. Costa Blanca Sports Science Week, 26-28 April 2018. Calpe. Alicante, Spain

\section{The effect of aerobic and anaerobic training with melatonin consumption on the expression of apoptotic genes BAX and BCL2 myocardial in rats after ischemic reperfusion}

\author{
HAMED ALIZADEH PAHLAVANI $\triangle$, ALI VEISI \\ Behbahan Faculty of Medical Sciences, Behbahan, Iran
}

\begin{abstract}
The present research aims to examine The Effect of aerobic and anaerobic training with melatonin consumption on the expression of apoptotic genes BAX and BCL2 myocardial in rats after ischemic reperfusion. In this study, Wistar rats $(n=38)$ were used weighing approximately 200-250 $\mathrm{g}$ with two to three months old. The study was conducted using Stroke $(n=7)$ and healthy $(n=7)$ rats to determine the stroke of isoprenaline injection; and left ventricular stroke was confirmed after the isoprenaline injection with the Terry Chromasone technique. Then, the rats were divided into 6 groups of aerobic $(n=4)$, anaerobic $(n=4)$, aerobic with melatonin $(n=4)$, anaerobic with melatonin $(n=4)$, Melatonin $(n=4)$ and control group $(n=4)$. The research group rats were familiar running on treadmill for a week, and One-month aerobic and anaerobic training was performed with three sessions per week. Also, melatonin gavage $(10 \mathrm{mg} / \mathrm{kg})$ was applied to melatonin treatment groups. After two rest days, all rats were injected with isoprenaline at 24 hours intervals. However, control group rats were only injected with isoprenaline. Data was analyzed using independent $T$, one way ANOVA and 2- $\Delta \Delta \mathrm{ct}$ formula. The results of this study showed that aerobic and anaerobic training and melatonin alone had a negligible effect on BAX and BCL2 genes expression, However, aerobic and anaerobic training combined with melatonin was able to reduce and increase the expression of the BAX and BCL2 genes, respectively. Of course, these changes had a significant effect on melatonin alone. Therefore, it seems that this intensity and duration of training with melatonin intake into melatonin alone can provide the
\end{abstract}

Corresponding author. Behbahan Faculty of Medical Sciences, Behbahan, Iran.

E-mail: Alizadehh76@yahoo.com

Supplementary Issue: Spring Conferences of Sports Science. Costa Blanca Sports Science Week, 26-28 April 2018. Calpe. Alicante, Spain.

JOURNAL OF HUMAN SPORT \& EXERCISE ISSN 1988-5202

(c) Faculty of Education. University of Alicante

doi: 10.14198/jhse.2018.13.Proc2.29 
anti-apoptotic routes of training to minimize the effects of acute stroke. Key words: AEROBIC TRAINING, ANAEROBIC TRAINING, MELATONIN, BAX, BCL-2.

\section{Cite this article as:}

Alizadeh Pahlavani, H., \& Veisi, A. (2018). The effect of aerobic and anaerobic training with melatonin consumption on the expression of apoptotic genes BAX and BCL2 myocardial in rats after ischemic reperfusion. Journal of Human Sport and Exercise, 13(2proc), S454-S466. doi:https://doi.org/10.14198/ihse.2018.13.Proc2.29 


\section{INTRODUCTION}

Before 1900, infectious diseases and malnutrition were the most common causes of death in the world. Now, cardiovascular diseases (CVDs) are the most common causes of death in many parts of the world. If such a situation continues in the same way, death rate can be increased in the future by considering age. Of course, it is predicted that by 2020, number of deaths caused by cardio-hereditary diseases (CHDs) is the most important cause of death. According to research reports, accumulation of lipoproteins, endothelial damage and inflammation are some of multiple processes that are involved to initiate and progress atherosclerosis (Longo et al, 2012). However, hypoxia is one of the causes of damaging heart muscle cells and the programmed heart cell death called apoptosis; so that cells and mitochondria of an area will be inflated, lose their membrane integrity, as a result, in-cell material such as pre-apoptotic proteins are released that cause inflammatory reactions and intensify initial injuries. In contrast, anti-apoptotic proteins can reduce cell death caused by incentives through anti-apoptotic activity and influencing caspases. At the same time, Bax preapoptotic protein and $\mathrm{Bcl}-2$ anti-apoptotic protein are the most important involved proteins in apoptosis, which they used as a measure to reduce or increase cell death. So any factor that can increase Bax/Bcl-2 ratio, it can be effective in reducing apoptosis (Shariatzadeh et al, 2008). However, several factors can reduce the severity of coronary artery lesions and myocardial apoptosis that physical activity is the most important one because the risk of developing coronary artery disease in ready or active adults is $30-40 \%$ lower than inactive individuals (Longo et al, 2012). Other studies showed that aerobic exercise increases anti-apoptotic factors such as Bcl-2 and decreases apoptotic factors such as Bax in myocardium (Santana et al, 2014). It seems, reducing apoptosis markers such as Bax and Bad and increasing anti-apoptotic markers such as Bcl-2 in myocardial cells are the possible causes of exercise benefits to protect the heart. In this regard, a study showed that exercise activities reduced increasing ratio $\mathrm{Bax} / \mathrm{Bcl}-2$ ratio, apoptosis and cardiac remodeling in old rats (Bum Kwak et al, 2006). Another study indicated that the Bax/Bcl-2 ratio in endurance training group was more than the control and acute exercise groups (Delchev et al, 2005). Finally, another research pointed out that exercise activity can increase protein expression of heart protection such as $\mathrm{Bcl}-2$ and $\mathrm{HSP}_{72}$ in rats with blood pressure (Lajoie et al, 2004). Therefore, we can say that regular aerobic exercise program can improve aerobic capacity of coronary patients. Versus, Jafary et al showed aerobic training for 12 week has no significant effect on Bcl-2 gene expression. In this regard, research showed that exercise group alone or with vitamin $E$ and lipoic acid alpha has no significant effect on bax, bcl2 genes and bcl2 / bax ratio than the non-complementary group. Of course, this study showed that the bax gene in the trained and supplement group was reduced in comparison with the non-supplementary training group (marsh et al, 2005).Another study showed that BAD apoptosis gene did not decrease after 13 weeks of exercise (Santana et al, 2014). In general, the relation between apoptosis and regular exercise program can be due to the lack of intermediate oxygenation, an increase in oxidative and inflammatory factors during exercise; So that the continuation of physical activity leads to increased cardiac output, peripheral oxygen removal, increased antioxidant activity, decreased inflammatory factors, and improved aerobic capacity and ultimately increases resistance to cell death. Of course, more physical activity would be more beneficial (sagiv, 2012). In other words, people who maintain a regular physical activity program for a longer or more intense period will probably get more benefits than those who have less physical activity (Pescatello, 2014). On the other hand, long-term medications such as beta-blockers, melatonin, aspirin, plavix, warfarin and statin have been considered. The consumption of these substances is due to the role of vascular dilatation. Also, The occurrence of a stroke in the morning has been reported due to decreased secretion of melatonin in the body. In confirmation of this issue, people receiving oral melatonin reduce blood pressure in normal blood samples. It has also been reported that levels of melatonin are reduced in people with stroke and cardiovascular disease. Finally, the finding that melatonin receptors exist in human arteries expresses the direct role of the hormone in controlling the localized diameter of the blood vessels (Farhud et al, 2008). Meanwhile, new 
studies have pointed to the antioxidant, anti-inflammatory, and anti-apoptotic properties of melatonin. In this regard, a study showed that melatonin reduces the ratio of mitochondrial oxygen consumption, mitochondrial complex I and II activity, the production of hydrogen peroxide, lipid peroxidase levels, cardiolipin levels, and cardiolipin oxidation (Petrosillo et al, 2006). Another study mentioned that melatonin has a protective effect of the heart through the removal of free radicals directly and indirectly through its antioxidant activity. A study in this regard showed that administration of melatonin $(10 \mathrm{mg})$ has significant protective effects on isoproterenol-induced infarction by adjusting exogenous antioxidant activity (Patel et al, 2010). Finally, the study concluded that melatonin decreases and increases Bax and Bcl2 (Forman et al, 2010). Overall, It seems that regular exercise and melatonin administration can improve the anaerobic and antioxidant capacity of coronary patients and also affect the reduction of apoptotic markers. Given that some people are susceptible to myocardial infarction and do not have enough time to practice regular exercises, they can decrease the incidence of infarction by increasing the intensity of exercise and reducing time through an anaerobic exercise by taking melatonin. Given that some studies have examined apoptotic and anti-apoptotic proteins (and not gene expression), others have reported inconsistent results with regard to the intensity and duration of the training; Also, some people have limited time to exercise, Can it be achieved with increasing intensity and low duration to the same results with moderate intensity and long duration ? The present study tries to investigate the effect of aerobic and anaerobic training with Consumption melatonin for 4 weeks on bax and bcl2 gene expression to reduce apoptosis in Ischemic-tolerated rats. Another aim of the present study was to compare two different intensities of aerobic and anaerobic training with consumption melatonin to reduce stroke.

\section{PROCEDURE}

In this study, Wistar rats $(n=38)$ were used weighing approximately $200-250 \mathrm{~g}$ with two to three months old. The study was conducted using Stroke $(n=7)$ and healthy $(n=7)$ rats to determine the stroke of isoprenaline injection (150 and $125 \mathrm{mg} / \mathrm{kg}$ ); and left ventricular stroke was confirmed after the isoprenaline injection with the Terry Chromasone technique. Then, the rats were divided into 6 groups of aerobic $(n=4)$, anaerobic $(n$ $=4)$, aerobic with melatonin $(n=4)$, anaerobic with melatonin $(n=4)$, Melatonin $(n=4)$ and control group $(n$ $=4)$. However, rats in aerobic $(n=4)$ and anaerobic $(n=4)$, aerobic with melatonin $(n=4)$, anaerobic with melatonin $(n=4)$ groups were familiar running on treadmill for a week, then they were exposed for one month training course with frequency of three times weekly (Høydal et al, 2007) (Table 1). Also, melatonin gavage (10 mg / kg) was applied to melatonin treatment groups. within 24 hours, all rats were injected isoprenaline with dose of 150 and $125 \mathrm{mg}$ per $\mathrm{kg}$ of their body. then, It was determined this dose drug caused fibrosis in the guidance group. Because Independent $t$ test showed the severity of fibrosis obtained from the $j$ image software was significantly different in the groups of isoprenaline and healthy rats, So that the fibrosis of the stroke group was more than healthy group (Table 3).

Table 1. Aerobic and anaerobic exercise protocol

\begin{tabular}{lcccc}
\hline \multicolumn{1}{c}{ Exercise process } & Heat & Aerobic exercise & Anaerobic exercise & Cool \\
Exercise components & 6 & 30 & $15-23$ & 6 \\
\hline Exercise time $(\mathrm{min})$ & $50-60 \%$ & $70 / 75 \%$ & $85-100 \%$ & $50-60 \%$ \\
\hline Exercise severity $\left(\mathrm{VO}_{2 \mathrm{max}}\right)$ & $15-20$ & $23-25$ & $30-38$ & $15-20$ \\
\hline Speed $(\mathrm{m} / \mathrm{min})$ & $90-120$ & $690-750$ & $690-750$ & $90-120$ \\
\hline Distance $(\mathrm{m})$ & 0 & $5-20$ & $5-20$ & 0 \\
\hline Treadmill gradient (degree) & & & & \\
\hline
\end{tabular}




\section{RNA Extraction}

For surgery, rats were anesthetized with chloroform. Then left ventricle of their heart was removed and rinsed with saline serum. Then they were placed in micro tubes and liquid nitrogen capsule for freezing. Samples of left ventricular were taken out from the nitrogen cylinders for homogenization. Then samples were extracted using manual protocols of RNA trisol. The extracted solution containing RNA was held in the freezer with -80 ${ }^{\circ} \mathrm{C}$ until cDNA synthesis. thenc DNA was synthesized using the Vivantis kit. In the following, there were obtained $20 \mathrm{ml}$ cDNA solution that was diluted with ratio of $1: 5$. It was transferred to the freezer with $-80^{\circ} \mathrm{C}$ or $-20^{\circ} \mathrm{C}$.

In order to find the frequency of primers, there were found articles in this field and there were evaluated the frequency of Bax and Bcl-2 genes as well as beta-actin (Gorji et al, 2013; Shi et al, 2016; Yang et al, 2006). Then they were examined through ncbi website (Table 2).

Table 2. Frequency of the used primers in real-time

\begin{tabular}{|c|c|c|c|}
\hline Optimum temperature & Primer frequency & & he required gene \\
\hline \multirow[b]{2}{*}{60} & CCAGGACGCATCCACCAAGAAGC & $\mathrm{F}$ & \multirow[b]{2}{*}{ Bax } \\
\hline & TGCCACACGGAAGAAGACCTCTCG & $\mathrm{R}$ & \\
\hline \multirow{2}{*}{60} & CAGAGATGTCCAGTCAGCTG & $\mathrm{F}$ & \multirow{2}{*}{ Bcl-2 } \\
\hline & CAGTCATCCACAGAGCGATG & $\mathrm{R}$ & \\
\hline \multirow{2}{*}{60} & CTAAGGCCAACCGTGAAAAGATG & $\mathrm{F}$ & \multirow{2}{*}{ Beta-actin } \\
\hline & TGGTACGACCAGAGGCATACAG & $\mathrm{R}$ & \\
\hline
\end{tabular}

\section{Real-Time Preparation}

In order to prepare materials of real-time, there were poured 4 lambda cDNA from each research team into wells of real-time device. Then there were added 6 lambda of master mix solution $(0.5$ lambda of the forward primer and rivers, 0.5 lambda water and 5 lambda master mix) to it. After that, the samples were vortex and then they were run for 5 minutes at $95^{\circ} \mathrm{C}$ (initial formation), 10 seconds at $95^{\circ} \mathrm{C}$ (secondary formation), 15 seconds at $60^{\circ} \mathrm{C}$ (primers' connection) and 20 seconds at $72{ }^{\circ} \mathrm{C}$ (replication). After the second stage, the reaction was repeated for 40 cycles. The ct-related reactions were extracted and recorded by software.

\section{Statistical Method}

The independent t-test was firstly used to examine mean difference of fibrosis in Stroke and healthy groups. Next, there was used 2- $\Delta \Delta c t$ formula to quantify expression levels of the considered genes. Then the fold change formula was used for multiple gene expression. Also, the ANOVA test was used to examine differences in gene expression between the groups. Finally, there was considered 0.05 as the significance level for all tests.

\section{RESULTS}

These results indicate that injection of isoprenaline with 150 and $125 \mathrm{mg} / \mathrm{kg}$ of body weight for two consecutive days has the potential to cause fibrosis in the heart of the rats, indicating a heart attack (Table 3) (Fig. 1). 
Table 3. Fibrosis amount in the pilot group

\begin{tabular}{ccccccc}
\hline & Statistical indicator & & & & \\
Group & & Number & Fibrosis mean & SD & Loon & Sig. \\
\cline { 1 - 5 } stroke & 7 & 107.85 & 11.95 & & \multirow{2}{*}{0.098} & \multirow{2}{*}{0.00} \\
\hline Healthy & 7 & 24.57 & 17.17 & & \\
\hline
\end{tabular}

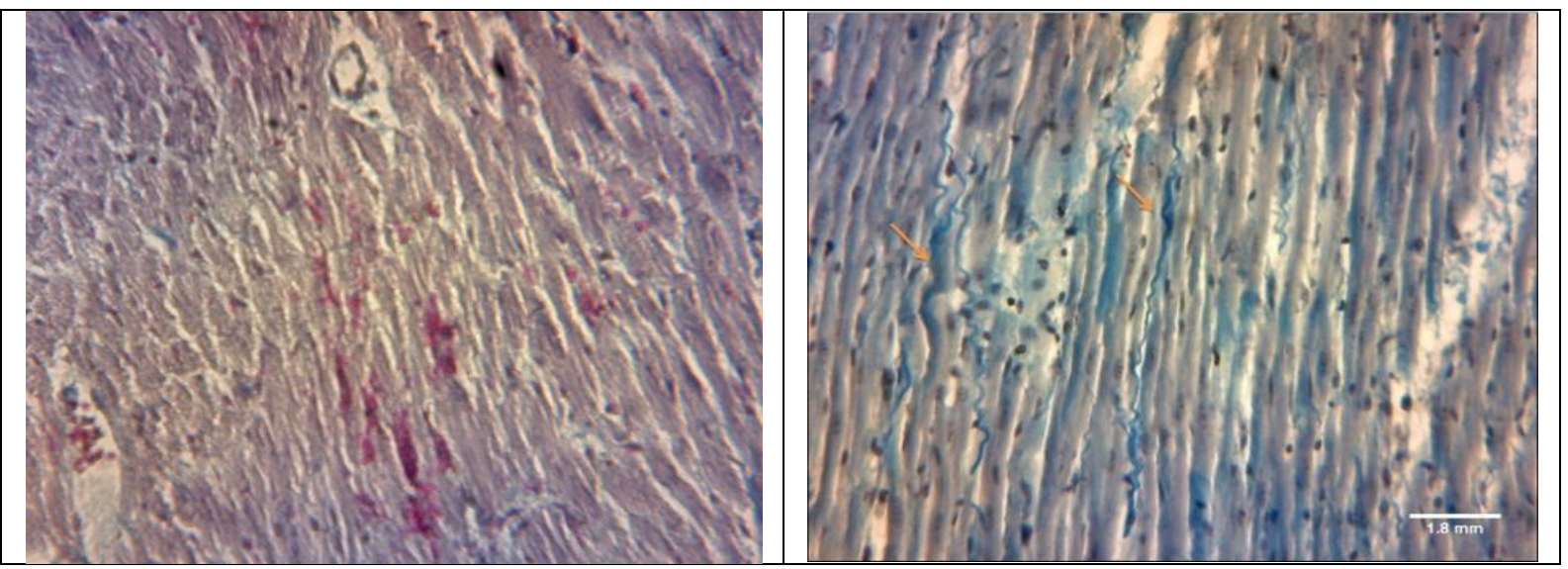

Figure 1. Images of isoprenalin-induced and healthy groups

The results of study showed that BAX gene expression in melatonin, aerobic and anaerobic, aerobic with melatonin and anaerobic with melatonin group Had decreased to about 1.67, 0.87, 0.9, 0.51 and 0.52 equal, respectively. In contrast, the expression level of BCL2 gene in the melatonin, aerobic and anaerobic, aerobic with melatonin and anaerobic with melatonin group increased to about 1.26, 1.63, 3.39, 1.64, and 2.35 equal, respectively (figure 2).

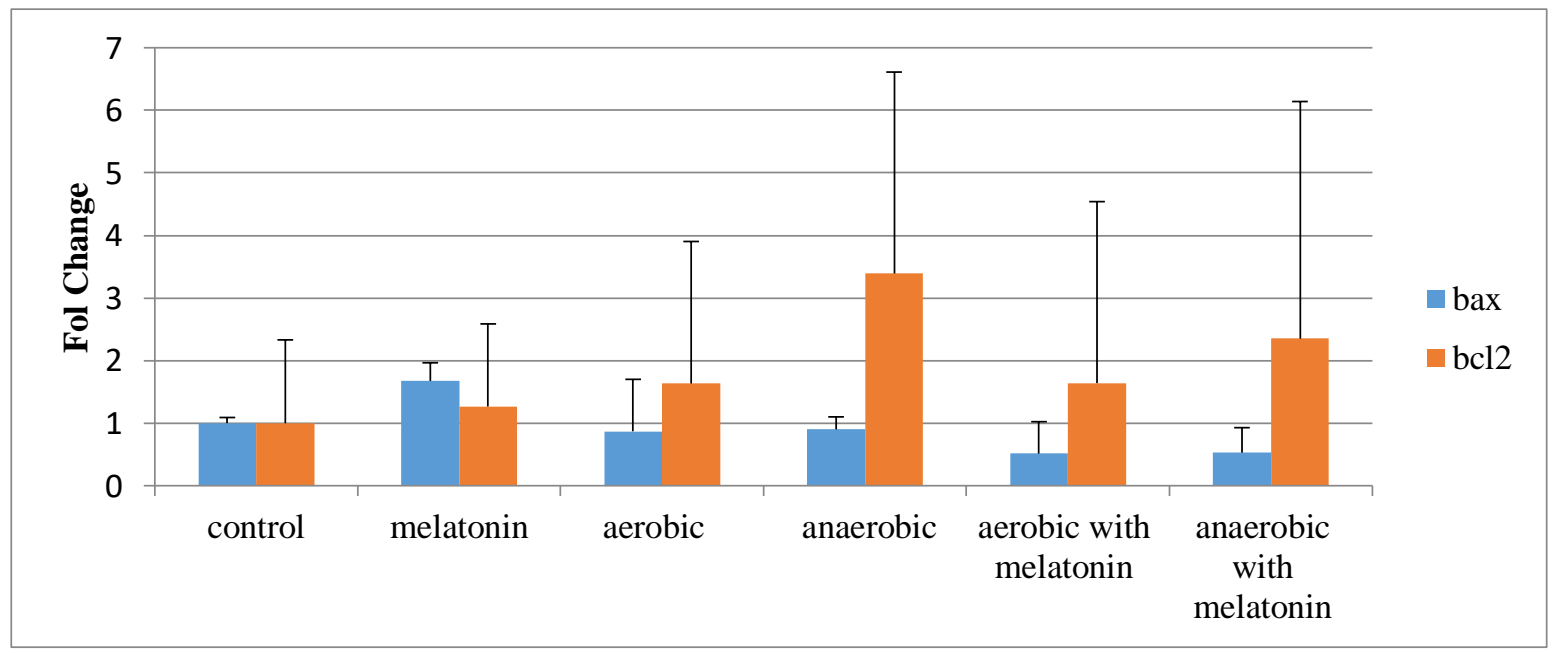

Figure 2. The Bax and Bcl-2 gene expression in the research groups

To evaluate the significance levels, there were used the values of 2- $\Delta \Delta \mathrm{ct}$. Meanwhile, the independent t-test was used to compare groups (Table 4). The results of Table 3 show that the expression of bax gene was only significant in aerobic with melatonin and anaerobic with melatonin groupsm; Because the bax value was 
reported ( $p=0.02$ and 0.02 respectively), which is smaller than the significant value of 0.05 ; But in other groups there were no significant changes. However, as seen in the fold change chart, the expression of bax and bcl2 genes decreased and increased with aerobic and anaerobic training compared with the control group.

Table 4. Comparing Bax and $\mathrm{Bcl}-2$ genes in research groups

\begin{tabular}{|c|c|c|c|c|c|}
\hline \multicolumn{2}{|r|}{ BCL2 } & \multicolumn{2}{|r|}{ Bax } & \multirow[b]{2}{*}{ groups } & \multirow[b]{2}{*}{ groups } \\
\hline Sig & $\begin{array}{c}\text { difference in } \\
\text { averages }\end{array}$ & Sig & $\begin{array}{c}\text { difference in } \\
\text { averages }\end{array}$ & & \\
\hline 1 & $-0 / 26$ & $0 / 34$ & $-0 / 66$ & melatonin & \multirow{5}{*}{ control } \\
\hline $0 / 99$ & $-0 / 63$ & $0 / 99$ & $0 / 12$ & aerobic & \\
\hline $0 / 79$ & $-2 / 39$ & 1 & $0 / 1$ & anaerobic & \\
\hline $0 / 99$ & $-0 / 64$ & $0 / 65$ & $0 / 49$ & aerobic with melatonin & \\
\hline 0/97 & $-1 / 35$ & $0 / 67$ & $0 / 48$ & $\begin{array}{l}\text { anaerobic with } \\
\text { melatonin }\end{array}$ & \\
\hline 1 & $0 / 26$ & $0 / 34$ & $0 / 66$ & control & \multirow{5}{*}{ melatonin } \\
\hline 1 & $-0 / 37$ & $0 / 19$ & $0 / 79$ & aerobic & \\
\hline $0 / 85$ & $-2 / 13$ & $0 / 21$ & $0 / 76$ & anaerobic & \\
\hline 1 & $-0 / 37$ & $0 / 02$ & $-1 / 16$ & aerobic with melatonin & \\
\hline 0/99 & $-1 / 09$ & $0 / 02$ & $-1 / 14$ & $\begin{array}{c}\text { anaerobic with } \\
\text { melatonin }\end{array}$ & \\
\hline $0 / 99$ & $0 / 63$ & $0 / 99$ & $-0 / 12$ & control & \multirow{5}{*}{ Aerobic } \\
\hline 1 & $0 / 37$ & $0 / 19$ & $-0 / 79$ & melatonin & \\
\hline $0 / 93$ & $-1 / 76$ & 1 & $-0 / 02$ & anaerobic & \\
\hline 1 & $-0 / 00$ & $0 / 86$ & $0 / 36$ & aerobic with melatonin & \\
\hline 0/99 & $-0 / 72$ & $0 / 87$ & $0 / 35$ & $\begin{array}{l}\text { anaerobic with } \\
\text { melatonin }\end{array}$ & \\
\hline $0 / 79$ & $2 / 39$ & 1 & -1 & Control & \multirow{5}{*}{ Anaerobic } \\
\hline $0 / 85$ & $2 / 13$ & $0 / 21$ & $-0 / 76$ & Melatonin & \\
\hline $0 / 93$ & $1 / 76$ & 1 & $0 / 02$ & Aerobic & \\
\hline $1 / 72$ & $0 / 93$ & $0 / 82$ & $0 / 39$ & aerobic with melatonin & \\
\hline 0/99 & $1 / 03$ & $0 / 84$ & $0 / 38$ & $\begin{array}{l}\text { anaerobic with } \\
\text { melatonin }\end{array}$ & \\
\hline $0 / 99$ & $0 / 64$ & $0 / 65$ & $-0 / 49$ & Control & \multirow{5}{*}{ aerobic with melatonin } \\
\hline 1 & $0 / 37$ & $0 / 02$ & $-0 / 116$ & melatonin & \\
\hline 1 & $0 / 00$ & $0 / 86$ & $-0 / 36$ & aerobic & \\
\hline $0 / 93$ & $-1 / 75$ & $0 / 82$ & $-0 / 39$ & anaerobic & \\
\hline 0/99 & $-0 / 71$ & 1 & $-0 / 01$ & $\begin{array}{l}\text { anaerobic with } \\
\text { melatonin }\end{array}$ & \\
\hline $0 / 97$ & $1 / 35$ & $0 / 68$ & $-0 / 48$ & Control & \multirow{5}{*}{$\begin{array}{l}\text { anaerobic with } \\
\text { melatonin }\end{array}$} \\
\hline $0 / 99$ & $1 / 09$ & $0 / 02$ & $-1 / 14$ & melatonin & \\
\hline $0 / 99$ & $0 / 72$ & $0 / 87$ & $-0 / 35$ & aerobic & \\
\hline $0 / 99$ & $1 / 03$ & $0 / 84$ & $-0 / 38$ & anaerobic & \\
\hline $0 / 99$ & $0 / 71$ & 1 & $0 / 01$ & aerobic with melatonin & \\
\hline
\end{tabular}

\section{DISCUSSION AND CONCLUSION}

Findings from the pilot study showed that injection of isoprenaline with 150 and $125 \mathrm{mg} / \mathrm{kg}$ body weight can cause fibrosis in the myocardium. In this regard, studies have highlighted the pathway for developing 
myocardial low-oxygenation with isoprenaline induction, such as increasing lipid peroxidation, increasing free radicals, decreasing exogenous antioxidants, increasing lipid accumulation, increasing stimulation of myocardial $\beta 1$ receptor, increasing intracellular calcium and necrosis with Inflammation; all of which can lead to damage to the cell membrane. Ultimately, cell membrane damage results in the release of intracellular enzymes and myocardial damage (Vibha et al, 2011). In support of this research, the effect of isoprenaline derivatives Showed that isoproterenol causes an increase in intracellular calcium from catecholamines and an increase in calcium intake from the L-type calcium channel. Then, The cytosolic and mitochondrial calcium is further enhanced and leads to a decrease in plasma calcium, and the receptor sensitive to calcium of parathyroid gland is stimulated and parathyroid hormone secretion is activated. This hormone helps further increment intracellular calcium and excessive accumulation of calcium leads to low oxygen levels in organelles and mitochondrial permeability. Osmotic swelling and organelles decomposition with the absence of ATP causes necrosis and fibrosis and myocardial injury, in next, ROS will help with these events (Shahbaz et al, 2011). Also, when cardiac reperfusion ischemic injury are imposed factors such as, excessive production of ROS, increased intracellular calcium, $\mathrm{H}$ leakage in mitochondria levels and inflammation Which leads to the opening of permeable mitochondrial pores. This can lead to reduced ATP, irreversible oxidation of protein, fat and DNA in cardiomyocytes, and begin the process of apoptosis (Jin Ha et al, 2010). Because, oxidation eventually results in the displacement of base in DNA, such as the conversion of AT to CG and GC into TA, causing transversal mutations (Cooke et al, 2003). In contrast, research has shown that exercise improves systolic myocardial function in mice with infarction, Which results from modulating the expression of intracellular calcium signaling proteins such as calsequestrin and L-type calcium channel (Guizoni et al, 2016). In confirmation of the above, another study showed that regardless of intensity, volume and type of exercise, the antioxidant and pre-oxidant parameters tended to increase and decrease after physical exercise (de Sousa et al, 2017). All of these indicate myocardial protection against infarction resulting from exercise.

The results of this study showed that the use of melatonin increases the expression of apoptotic genes; and this finding is incompatible with the findings of study by Forman et al (Forman et al, 2010). Because the researchers studied the beneficial effects of melatonin on changing the heart of old rats, While the current study has used 2 to 3-month-old rats; And it seems that in the study of Forman et al, melatonin has often used its antioxidant role, Because antioxidants are reduced in older rats, and Subsequently, this reduction is compensated by melatonin treatment, and can reduce the amount of apoptosis and its markers, such as bax and bad. While in the present study, melatonin has often used sleeping and vascular dilatation role in 2 to 3 months old rats; So that the level of penetration of fat in the myocardium is increased with sleep and inactivity and dilation of the vessels, and Inflammatory indices such as TNF-a and free radicals such as ROS and RNS are further increased; and ultimately, the myocardium is susceptible to ischemic injury. Therefore, It seems that melatonin can play a double role with its own properties, such as antioxidant, vasodilatation, and sleeping properties; So that it can play a useful role if its antioxidant properties prevail; But if the role of vascular dilatation and sleeping prevails, it can play a detrimental role.

It also seems that the reason for the increasing of bcl2 is that Redox-sensitive compounds are involved in signaling of cell protection and leads to the transcription response of AP-1 and NrF2. With these pathways, melatonin stimulates antioxidant genes and toxication excretion, and in turn acts as an enhancer of the glutathione system. Ultimately, melatonin decreases the production of reactive oxygen species and activity of anti-apoptotic agents such as bcl2 and sensitive to redox (Luchetti et al, 2010). On the other hand, Heidari et al noted that the high production of free radicals and lipid peroxidation would disrupt the building of the lipid membrane and other cellular compositions. The researchers concluded from their findings that the use of melatonin prevents changes in fat profiles and increased MDA resulting from a high fructose diet in the heart tissue (Heidari et al, 2011). Because this hormone, after passing through the biological membrane, 
stimulates the expression of antioxidant genes and inhibits the pro-oxidant genes, and thus increases the antioxidant capacity of the cells (Hardeland, 2005). Another possible reason for the increase of the BCL2 gene is the reduction of adrenalin and subsequently the maintenance of calcium homeostasis, which leads to the myocardial dilatation; Consequently, it causes the least disturbance and permeability of the mitochondria in the cell. Therefore, the antioxidant property of melatonin decreases iNOS and oxidants as well as increases NRF2 and JAK2 / STAT3 pathways, which both inhibit inflammation and oxidation of the myocardium (Luchetti et al, 2011). On the other hand, the lack of mobility of the melatonin group, may lead to an increase in adipokines such as visfatin within the plasma; Which ultimately increases myocardial inflammation and oxidative and the myocardium makes more susceptible to ischemic injury. At the same time, when oxidative agents and lipid permeation predominate over antioxidant agents and lipid oxidation, they can play a damaging role in the myocardium. It seems that melatonin can have multifactorial effects for heart reperfusion ischemic injury.

Findings of the present research showed that one month aerobic exercise will respectively decrease and increase Bax and Bcl-2 gene expression after ischemia reperfusion through isoprenaline, but they were not significant. Contrary to the results of this study, Kang et al Developed a physiological and pathological hypertrophy, respectively, with exercise and high-salt diets; and showed that exercise induces changes in the protein of the family bcl2, caspases, and increases the bcl2 / bax ratio. Lajoie et al (2004) showed that exercise training for 5 days per week at a speed of $18 \mathrm{~m} / \mathrm{min}$ for 120 minutes daily can increase hsp72 and Bcl-2 anti-apoptotic proteins. Delshev et al (2005) showed that Bcl-2 level had the most increase in endurance training for 8 weeks, compared to other research groups (Delshev et al, 2005). To support this research, Marfe et al (2012) showed that exercise training will reduce Bax and increase Bcl-2 by increasing SIRT1 and SIRT7 (Marfe et al, 2012). Bum Kwak et al (2013) demonstrated that exercise training can reduce Caspase9 as well as Bax/Bcl-2 ratio and eventually, it can decrease and increase Bax and Bcl-2 protein. In this regard, Santana et al (2014) showed that exercise training for 13 weeks increased mRNA and protein of antiapoptotic factors such as Bcl-2, Survivin, ILK and AKT (Santana et al, 2014). On the other hand, Jaafari et al (2015) showed that value of Bax gene and Bax/Bcl-2 ratio of exercise group was less than the control group. However, the study did not find significant differences in Bcl-2 genes between the two groups. Of course, there was reported higher level of Bcl-2 for the exercise group, compared to the control group. In another research, Chengji et al (2016) showed Bax and Bcl-2 gene expression was not significantly reduced in the aerobic exercise group with myocardial infarction, compared with Ml. Given these results, it seems that different aspects of the present and previous studies such as exercise duration can affect significance of Bax and $\mathrm{Bcl}-2$ gene expression because the more exercise duration, the better compatibility of practice and exercise useful aspects such as reducing fat lipoproteins and increasing antioxidants to oxidants factors and consequently, reducing inflammatory factors. because Most of studies have considered duration of 8 and 13 weeks to practice, while the present research examined duration of 4 weeks. On the other hand, some studies have proposed five exercise sessions weekly, but the present research has examined three exercise sessions weekly. In this regard, Lajoie et al were trained rats for 120 minuts per each session; but present research were trained rats for 42 and 23 minuts. Therefore, it can be said that with the least stimulus of training, three days a week and for 4 weeks of training with moderate and high intensity, can not decraesed myocardial infarction. On the other hand, oxidant, inflammatory, lipid and apoptotic factors such as Caspase 3 are reduced by training adaptation. In contrast, antioxidant agents, cellular pre-existing, such as the IGF1$\mathrm{R} / \mathrm{PI}$ KK / AKT pathway is increased, Which can generally reduce heart apoptosis.

In contrast, the results of this study showed one month anaerobic training were reduced and increased Bax and $\mathrm{Bcl}-2$ gene expression by isopernaline. It seems that training intensity can also affect the results because the more intensity of exercise, the more profit for body. Now it seems that if we increase number and time of 
exercise sessions weekly, we can get favorable results of decreasing and increasing Bax and Bcl-2 genes. As a result, we can observe reducing volume of cardiac apoptosis because factors such as reducing lipoproteins (LDL, VLDL and leptin), increasing antioxidants (glutathione and superoxide dismutase), decreasing inflammatory factors such as TNF- $a, I L-6$ and free radicals as well as increasing growth factors such as IGF-1 and AKT and NFAT that are created by total compatibility of exercise can reduce volume of cardiac apoptosis, since they are associated with reducing cellular homeostasis disruptive factors such as caspases, calpain, ROS, RNS and increasing cell survival factors such as STATs, PI3K, PGC1-a, MEK and ERK.

In this regard, scott et al (2004) mentioned exercise training is protection versus reperfusion ischemia injury. Because endurance training protect all levels of myocard reperfusion ischemia Injury. This research exerciseinduced myocard protective mechanisms expressed increase in the coronary artery circulation, expression of ER stress proteins, increase the activity of cyclooxygenase-2, induction of heat shock proteins of myocard, increased myocardial cytosolic antioxidant capacity, increased signaling nitric oxide, changes in mitochondrial phenotypic, increase and changes in the ATPsensitive potassium channels expression and increase the mitochondrial inner membrane. Finally, the researchers showed increased antioxidant levels and increased myocardial expression of ATP-sensitive potassium channels assist cardiac protection against reperfusion ischemic injury. Because these factors reduce the mitochondrial damage and Increases the integrity of the mitochondrial membrane; however it increases the BCL2 gene expression in mitochondria.

Other findings of the study showed that anaerobic exercise with melatonin reduced and increased the expression of the bax and bcl2 genes. However, the reduction in bax gene was not significant in comparison with the control group, But it was expressed in comparison with the melatonin group. These findings indicate that aerobic exercise with melatonin has been able to reduce the expression of bax more than other groups; Because it seems that the use of antioxidant properties of melatonin has been able to help inappropriate training in the anaerobic training group. It seems that melatonin consumption with antioxidant properties has been able to help inappropriate training in the anaerobic training group and compensate antioxidant deficiencies. On the other hand, physical training has compensated lipid profiles such as LDL and VLDL in the melatonin group due to immobility and sleepiness Because these factors have synergistic effects, Which ultimately leads to a decrease in the amount of bax in the myocardium. Exercise and melatonin may also potentially improve calcium homeostasis, mitochondrial permeability, cytochrome $\mathrm{C}$ and adrenaline release; So that all these factors can interfere with the reduction of ischemic reperfusion injury. Finally, research showed that exercise and melatonin could increase myocardial survival due to increased survival factors such as IGF1-R / PI3K / AKT and JAK2 / STAT3. With regard to the above, it seems that a better result can be achieved by increasing the duration of exercise and the melatonin consumption.

Of course, the reason for the increased expression of $\mathrm{BCL} 2$ gene in the exercise group with melatonin versus exercise alone seems to be a lower level of inflammation in the exercise group with melatonin. Because Veneroso et al (2009) stated when the heart muscle undergoes acute exercise, it increases the symptoms of cell damage. Because exercise is associated with a significant increase the activity of maloparoxidases and levels of TNF- $a, I L-1$, and IL-6 mRNA. In addition, the researchers showed that the concentration of mRNA and protein of the intracellular adhesion molecule, Inducible nitric oxide synthase and cyclooxygenase 2 increased. As well as, Significant activity of NF-KB was observed in the training rats. In contrast, these effects were generally blocked by melatonin treatment. Therefore, it can be said that melatonin protects from exercise-induced cardiovascular injury. In confirmation of this, Kumar et al (2002) showed that in subjects after exercise, lipid peroxidation products such as malonyl dialdehyde increased significantly. While superoxide dismutase and glutathione peroxidase were decreased. Also, the base of lipid peroxidation was 
significantly reduced in melatonin-treated patients compared to melatonin-free study. Subsequently, After exercise, superoxide dismutase and glutathione peroxidase were reduced after treatment with melatonin. In confirmation of the above, Santos et al (2013) showed that trained rats with Stanzelol injection experienced an increase in blood pressure and relative weight of the heart; So that the deviation of the left axis of the heart increased. Of course, although melatonin did not prevent cardiac hypertrophy in rat with Stanzelol injection; But blood pressure and cardiac catalase activity were maintained, and prevented the deviation of the electrical axis of the heart from Stanzelol. This finding showed that chronic administration of Stanzelol causes cardiovascular side effects; Which is partially reduced by melatonin treatment. The researchers noted that the combination of melatonin and exercise can minimize the side effects of stanzolol in the cardiovascular system.

Finally, this study suggest aerobic and anaerobic exercise for 4 weeks cant reduce volume of heart-failure in patients prone to ischemic coronary artery; But given that the improvement in anaerobic exercise has been better, it seems to increase the duration and intensity of exercise can reduce the size of a possible stroke. Therefore, the present research suggests that patients with coronary artery ischemic should implement longterm aerobic and anaerobic exercise to reduce risk of heart attack and myocardial infarction.

\section{ACKNOWLEDGMENTS}

This article is the result of a research work at the Kharazmi University Cellular and Molecular Biology Laboratory, and thanks for the great cooperation of this collection.

\section{REFERENCES}

F, L., K. h, and L. j, Harrison's Principles of Internal Medicine. New York, NY: McGraw-Hill Professional, 2012. 18th ed.

M, S., Apoptosis programmed cell death. Arak, arak Univ Press, 2009. 1th ed.

Santana, E.T., et al., Aerobic exercise training induces an anti-apoptotic milieu in myocardial tissue. Motriz: Revista de Educação Física, 2014. 20: p. 233-238. https://doi.org/10.1590/S198065742014000200015

Kwak, H.-B., W. Song, and J.M. Lawler, Exercise training attenuates age-induced elevation in Bax/Bcl-2 ratio, apoptosis, and remodeling in the rat heart. The FASEB Journal, 2006. 20(6): p. 791-793. https://doi.org/10.1096/fi.05-5116fje

Delchev, S., et al., Bcl-2/Bax ratio, mitochondrial membranes and aerobic enzyme activity in cardiomyocytes of rats after submaximal training. Vol. 48. 2006. 50-6.

Lajoie, C., A. Calderone, and L. Béliveau, Exercise training enhanced the expression of myocardial proteins related to cell protection in spontaneously hypertensive rats. Pflügers Archiv, 2004 . 449(1): p. 26-32. https://doi.org/10.1007/s00424-004-1307-0

Jafari, A., et al., Effect of Exercise Training on Bcl-2 and Bax Gene Expression in the Rat Heart. Gene Cell Tissue, 2015.

Marsh, S.A., et al., Bcl-2 in endothelial cells is increased by vitamin $\mathrm{E}$ and a-lipoic acid supplementation but not exercise training. Journal of Molecular and Cellular Cardiology, 2005. 38(3): p. 445-451. https://doi.org/10.1016/j.yjmcc.2004.11.026

Sagiv, M., Exercise cardiopulmonary function in cardiac patients. Springer-Verlag London, 2012. https://doi.org/10.1007/978-1-4471-2888-5

Pescatello, L.S., ACSM's guidelines for exercise testing and prescription. Wolters Kluwer/Lippincott Williams \& Wilkins Health, 2014. 
Farhud, D. and L. Yazdanpanah, Glucose-6-phosphate dehydrogenase (G6PD) Deficiency. Iranian journal of public health, 2008. 37(4): p. 1-18.

Petrosillo, G., et al., Protective effect of melatonin against mitochondrial dysfunction associated with cardiac ischemia-reperfusion: role of cardiolipin. The FASEB journal, 2006. 20(2): p. 269-276. https://doi.org/10.1096/fi.05-4692com

Patel, V., et al., Cardioprotective effect of melatonin against isoproterenol induced myocardial infarction in rats: a biochemical, electrocardiographic and histoarchitectural evaluation. European journal of pharmacology, 2010. 644(1): p. 160-168. https://doi.org/10.1016/j.ejphar.2010.06.065

Forman, K., et al., Beneficial effects of melatonin on cardiological alterations in a murine model of accelerated aging. Journal of pineal research, 2010. 49(3): p. 312-320. https://doi.org/10.1111/j.1600-079X.2010.00800.x

Azamianjazi, A., et al., The Combined Effect of Endurance Training and Atorvastatin on the Extent of Necrosis Damageand Fibrosis Tissue in Male Wistar Rats Heart after Experimental Myocardial Infarction. medilam, 2016. 23(7): p. 28-38.

Høydal, M.A., et al., Running speed and maximal oxygen uptake in rats and mice: practical implications for exercise training. European Journal of Cardiovascular Prevention \& Rehabilitation, 2007. 14(6): p. 753-760. https://doi.org/10.1097/HJR.0b013e3281eacef1

Vibha, L., et al., Protective Effect of Medicinal Garlic Against Isoprenaline Induced Myocardial Infarction in Rats. International Journal of Pharmacology, 2011. 7: p. 510-515. https://doi.org/10.3923/ijp.2011.510.515

Shahbaz, A.U., et al., Calcium and zinc dyshomeostasis during isoproterenol-induced acute stressor state. Am J Physiol Heart Circ Physiol, 2011. 300(2): p. H636-44. https://doi.org/10.1152/ajpheart.00900.2010

Jin Ha, S. and W. Weon Kim, Mechanism of Ischemia and Reperfusion Injury to the Heart:From the Viewpoint of Nitric Oxide and Mitochondria. Chonnam Medical JournaL, 2010. 46 (3): p. 129-139. https://doi.org/10.4068/cmi.2010.46.3.129

Cooke, M.S., et al., Oxidative DNA damage: mechanisms, mutation, and disease. Faseb j, 2003. 17(10): p. 1195-214. https://doi.org/10.1096/fi.02-0752rev

Guizoni, D.M., et al., Effects of late exercise on cardiac remodeling and myocardial calcium handling proteins in rats with moderate and large size myocardial infarction. Int J Cardiol, 2016. 221: p. 40612. https://doi.org/10.1016/j.ijcard.2016.07.072

de Sousa, C.V., et al., The Antioxidant Effect of Exercise: A Systematic Review and Meta-Analysis. Sports Med, 2017. 47(2): p. 277-293. https://doi.org/10.1007/s40279-016-0566-1

Luchetti, F., et al., Melatonin signaling and cell protection function. The FASEB Journal, 2010. 24(10): p. 3603-3624. https://doi.org/10.1096/fi.10-154450

Heidari, R. and V. Nejati, Protective Effects of Melatonin on Lipid Profile in Fructose Induced Dyslipidemia. Iranian Journal of Endocrinology and Metabolism, 2011. 13(4): p. 406-411.

Hardeland, R., Atioxidative protection by melatonin. Endocrine, 2005. 27(2): p. 119-130. https://doi.org/10.1385/ENDO:27:2:119

Kang, P.M., et al., Alterations in apoptosis regulatory factors during hypertrophy and heart failure. Am J Physiol Heart Circ Physiol, 2004. 287(1): p. H72-80. https://doi.org/10.1152/ajpheart.00556.2003

Marfe, G., et al., The modulation of sirtuins and apoptotic proteins in rats after exhaustive exercise. Open Journal of Molecular and Integrative Physiology, 2012. 2: p. 65-74. https://doi.org/10.4236/ojmip.2012.23010

Chengji, W. and H. Shoujun, Aerobic exercise can ameliorate heart function in patients with myocardial infarction through up-regulating M3 receptor. IJC Metabolic \& Endocrine, 2016. 13(Supplement C): p. 1-5. https://doi.org/10.1016/j.ijcme.2016.08.001 
Powers, S.K., J.C. Quindry, and A.N. Kavazis, Exercise-induced cardioprotection against myocardial ischemia-reperfusion injury. Free Radical Biology and Medicine, 2008. 44(2): p. 193-201. https://doi.org/10.1016/.jfreeradbiomed.2007.02.006

Veneroso, C., et al., Melatonin reduces cardiac inflammatory injury induced by acute exercise. Journal of pineal research, 2009. 47(2): p. 184-191. https://doi.org/10.1111/i.1600-079X.2009.00699.x

Kumar, K.V. and M. Naidu, Effect of oral melatonin on exercise-induced oxidant stress in healthy subjects. Indian Journal of Pharmacology, 2002. 34(4): p. 256-259.

dos Santos, G.B., et al., Melatonin reduces oxidative stress and cardiovascular changes induced by stanozolol in rats exposed to swimming exercise. The Eurasian journal of medicine, 2013. 45(3): $p$. 155.

\section{(c) (i) $\ominus$}

This title is licensed under a Creative Commons Attribution-NonCommercial-NoDerivs 4.0 Unported License. 